\title{
Ionizing Radiation Impacts on Cardiac Differentiation of Mouse Embryonic Stem Cells
}

\author{
Alexander Helm, ${ }^{1}$ Onetsine Arrizabalaga, ${ }^{1,2}$ Diana Pignalosa, ${ }^{1}$ \\ Insa S. Schroeder, ${ }^{1}$ Marco Durante, ${ }^{1,3}$ and Sylvia Ritter ${ }^{1}$
}

Little is known about the effects of ionizing radiation on the earliest stages of embryonic development although it is well recognized that ionizing radiation is a natural part of our environment and further exposure may occur due to medical applications. The current study addresses this issue using D3 mouse embryonic stem cells as a model system. Cells were irradiated with either X-rays or carbon ions representing sparsely and densely ionizing radiation and their effect on the differentiation of D3 cells into spontaneously contracting cardiomyocytes through embryoid body (EB) formation was measured. This study is the first to demonstrate that ionizing radiation impairs the formation of beating cardiomyocytes with carbon ions being more detrimental than X-rays. However, after prolonged culture time, the number of beating EBs derived from carbon ion irradiated cells almost reached control levels indicating that the surviving cells are still capable of developing along the cardiac lineage although with considerable delay. Reduced EB size, failure to downregulate pluripotency markers, and impaired expression of cardiac markers were identified as the cause of compromised cardiomyocyte formation. Dysregulation of cardiac differentiation was accompanied by alterations in the expression of endodermal and ectodermal markers that were more severe after carbon ion irradiation than after exposure to X-rays. In conclusion, our data show that carbon ion irradiation profoundly affects differentiation and thus may pose a higher risk to the early embryo than X-rays.

\section{Introduction}

$\mathrm{H}$ UMANS UNAVOIDABLY ARE EXPOSED to radiation that comprises both sparsely ionizing radiation (eg, $\gamma$ - or Xrays) and densely ionizing radiation (eg, $\alpha$-particles) [1]. Sparsely ionizing radiation is characterized by a more homogeneous dose distribution of relatively small energy depositions, whereas densely ionizing radiation exhibits a heterogeneous dose distribution with high local energy depositions. Its source may be natural [2] or artificial. Artificial sources used for medical purposes in diagnostics or therapy become increasingly important. For example, during cancer therapy, $\sim 50 \%$ of all patients with localized malignant tumors are treated with ionizing radiation [3]. Inevitably, such large patient cohorts will include pregnant women.

Since ionizing radiation poses a threat to the early embryo possibly leading to prenatal death, growth retardation, organ malformation, mental retardation, or childhood cancer [4], a thorough risk assessment of radiation effects is mandatory in situations of inevitable exposure of the conceptus in utero.
The United States Centers for Disease Control and Prevention recommends not exceeding an accumulative fetal radiation dose of $5 \mathrm{mGy}$ [5]. However, in average, an abdominal computed tomography scan results in a fetal dose of about 25 mGy [6]. Notably, even among radiology workers, the awareness and knowledge about radiation doses and risks is inadequate [7].

So far, data about radiation-induced biological effects during the earliest stages of human development are scarce, predominately stemming from atomic bomb survivors or observations made after fall-outs (eg, Chernobyl). Experimental in vitro data or animal studies point to a high radiosensitivity of the embryo throughout the whole prenatal period (for a summary, see Streffer et al. [8]), but the underlying mechanisms are poorly understood possibly leading to a suboptimal risk assessment. In this respect, pluripotent embryonic stem (ES) cells serve as an ideal in vitro model to elucidate the effects ionizing radiation may impose on the earliest steps of embryonic development. Derived from blastocysts, they grow indefinitely and, using

\footnotetext{
${ }^{1}$ Biophysics Department, GSI Helmholtz Centre for Heavy Ion Research, Darmstadt, Germany.

${ }^{2}$ IKERBASQUE, Basque Foundation for Science, Bilbao, Spain.

${ }^{3}$ Physics Department, Institute for Condensed Matter Physics, Technical University Darmstadt, Darmstadt, Germany.

(C) Alexander Helm, et al., 2015; Published by Mary Ann Liebert, Inc. This Open Access article is distributed under the terms of the Creative Commons Attribution Noncommercial License (http://creativecommons.org/licenses/by-nc/4.0/) which permits any noncommercial use, distribution, and reproduction in any medium, provided the original author(s) and the source are credited.
} 
appropriate protocols, can differentiate into cells of all three germ layers [9].

Differentiation of mouse ES cells can be initiated, among others, by the aggregation of the cells in embryoid bodies (EBs) using the hanging drop method [10] in the absence of leukemia inhibitory factor (LIF). As the heart is the first organ to develop, cardiac differentiation is the most meaningful measure to assess early embryo toxicity $[11,12]$. The generation of beating cardiomyocytes from mouse ES cells is well established $[13,14]$ and a particular rapid and predictive protocol using the hanging drop-based cardiac differentiation of D3 mouse ES cells for embryotoxicity screening is the embryonic stem cell test (EST). It was published as a standard operating procedure [15], successfully validated by the European Centre for the Validation of Alternative Methods, and is widely used in pharmacology [16].

In this study, we investigated the influence of sparsely ionizing X-ray and densely ionizing carbon (C)-ion irradiation that is used in state-of-the-art cancer therapies [17] on the cardiac differentiation of mouse D3 cells using a modified EST. Analyzing the number of EBs displaying beating cardiomyocytes, cell survival, the mRNA expression of mesodermal, and cardiac markers, we found that ionizing radiation decelerates cardiomyocyte formation. This phenomenon is most pronounced after C-ion irradiation. Even though control levels are reached after prolonged differentiation, hampered cardiac development as seen in vitro may be detrimental for the fetus. In addition, we analyzed the mRNA expression of endodermal and ectodermal markers. Again, the dysregulating effect of C-ions was more pronounced than that of X-rays supporting our notion that densely ionizing radiation poses a higher risk to the early embryo than sparsely ionizing radiation.

\section{Materials and Methods}

\section{Culture of undifferentiated ES cells}

D3 pluripotent mouse ES cells were purchased from ATCC (catalog number CRL-1934). Cells were maintained in gelatin (0.2\%)-coated tissue culture flasks in Dulbecco's modified Eagle's medium supplemented with $1 \%$ nonessential amino acids, $2 \mathrm{mM}$ L-glutamine, $1 \%$ penicillin/ streptomycin $(10,000 \mathrm{U} / \mathrm{mL}$ and $10,000 \mu \mathrm{g} / \mathrm{mL}$, respectively), $0.1 \mu \mathrm{M} \beta$-mercaptoethanol (Carl Roth $\mathrm{GmbH}+\mathrm{Co} . \mathrm{KG}), 18 \%$ heat inactivated fetal bovine serum, and $1,000 \mathrm{U} / \mathrm{mL} \mathrm{LIF}$ (Merck KGaA). Stock cultures were passaged every 2 or 3 days using a mixture of $0.1 \%$ trypsin and $0.2 \%$ ethylenediaminetetraacetic acid in phosphate-buffered saline without calcium and magnesium and reseeded at a density of $4 \times 10^{4}$ cells $/ \mathrm{cm}^{2}$ and $3 \times 10^{4}$ cells $/ \mathrm{cm}^{2}$, respectively. All cell culture products were purchased from Biochrom $\mathrm{GmbH}$ unless otherwise stated.

\section{Irradiation}

In all experiments, $0.5 \times 10^{6}$ cells were seeded into 12.5 $\mathrm{cm}^{2}$ tissue culture flasks. To study the direct radiation response of D3 cells, cultures were irradiated $48 \mathrm{~h}$ after seeding and processed immediately after exposure (in the following, referred to as 0 -h scheme). Moreover, the response of the progeny of irradiated cells was examined, that is, $72 \mathrm{~h}$ after exposure (in the following, referred to as 72 -h scheme). In the 72-h scheme, cells were exposed $24 \mathrm{~h}$ after seeding and passaged once ( $24 \mathrm{~h}$ post-irradiation). Both experimental schemes are displayed in the Supplementary Fig. S1 (Supplementary Data are available online at www.liebertpub.com/scd).

Cells in passage 10-14 were irradiated with either 1-6 Gy $\mathrm{X}$-rays or 0.5-3 Gy C-ions. For X-ray exposure, a Seifert Xray machine was used and operated at $250 \mathrm{kV}$ and $16 \mathrm{~mA}$ with a $1 \mathrm{~mm} \mathrm{Al}+1 \mathrm{~mm} \mathrm{Cu}$ filtering. The dose rate was about $2 \mathrm{~Gy} / \mathrm{min}$. Exposure to C-ions was performed at the heavy ion synchrotron SIS 18 (GSI Helmholtz Centre for Heavy Ion Research). A $25 \mathrm{~mm}$ spread-out Bragg peak was used, generated by active energy variation of the $\mathrm{C}$-ion beam in the range of 106-147 MeV/u, a condition typically applied in tumor therapy. The resulting dose-averaged mean linear energy transfer was $75 \mathrm{keV} / \mu \mathrm{m}$ compared to $2 \mathrm{keV} / \mu \mathrm{m}$ for $\mathrm{X}$-rays. The dose rate of C-ions was in the range of 1-3 Gy/ min. All exposures were done at room temperature and control samples were sham irradiated. Further details are given elsewhere $[18,19]$.

\section{Clonogenic cell survival}

Survival of cells was measured using the colony forming assay according to standard procedures [20]. Directly after exposure $(0 \mathrm{~h})$ or after a culture time of $72 \mathrm{~h}$, cells were trypsinized, counted, and 160 to $22 \times 10^{5}$ cells (depending on the applied dose), resulting in a statistically significant formation of at least 100 colonies, were plated in $25-\mathrm{cm}^{2}$ tissue culture flasks in triplicate. After 7 days of incubation in a medium containing LIF, cells were fixed and stained. Cell clusters consisting of at least 50 cells were counted as colonies using a stereomicroscope. In the present study, recently published data [18] were supplemented. Survival curves for $\mathrm{C}$-ions and $\mathrm{X}$-rays are the mean $\mathrm{X} \pm$ standard deviation (SD) of two and four experiments, respectively.

\section{Differentiation assay of ES cells}

To investigate whether irradiated D3 cells retain their ability to differentiate, cells in passage 10-14 were cultured in the absence of LIF applying the hanging drop method [15]. Briefly, cells were harvested 0 or $72 \mathrm{~h}$ postirradiation and hanging drops $(20 \mu \mathrm{L})$ generally consisting of 750 cells were formed. To assess to what extent the initial number of cells in the EBs determines the onset of cardiac differentiation, additional studies were performed in which hanging drops were generated comprising 500 or 850 cells. Hanging drop cultures were incubated for 3 days to enforce cell aggregation. Then, EBs were transferred to suspension culture. At day 5 of differentiation, single EBs were selected and seeded individually into the wells of gelatin $(0.2 \%)$-coated 24 -well plates.

\section{Evaluation of EB size and cardiomyocyte formation}

The diameter of EBs was microscopically determined at day 5 of differentiation when EBs were transferred to 24well plates. Measurements were performed at a Leica DMIL (50× magnification), using an eyepiece micrometer, or at the Nikon Eclipse TS100 (40× magnification), using the software NIS-Elements D (Nikon), both giving consistent results. Likewise, generation of contracting cardiomyocytes was microscopically evaluated (50× magnification; Leica DMIL). Measurements began 10 days after differentiation 
initiation, when beating clusters of cells appeared [15] and continued up to day 30 . Per dose and time point at least 48 EBs were analyzed and the cumulative fraction of EBs exhibiting contracting cells was determined.

\section{Apoptosis}

Apoptosis was measured in both undifferentiated pluripotent stem cells (ie, up to 3 days postirradiation) and their differentiating progeny (ie, EBs at day 1, 3, and 5). Apoptotic cells were identified by the typical morphological changes of the cell nucleus such as chromatin condensation or fragmentation [21,22]. Briefly, a single-cell suspension was prepared, and cells were fixed with $4 \%$ paraformaldehyde for $1 \mathrm{~h}$ at room temperature and stained with Hoechst $33342(0.8 \mu \mathrm{g} / \mathrm{mL})$ for $30 \mathrm{~min}$. The apoptotic index, that is, the ratio of the number of apoptotic cells to the total number of cells analyzed, was calculated by scoring 1,000 cells per dose and time point. Morphological analyses were validated in selected samples by immunocytochemical staining of cleaved caspase-3 (FITC Active Caspase-3 Apoptosis Kit; $\mathrm{BD}$ ) and subsequently analyzed using the flow cytometer FACSCanto II and the software FACSDiva (both BD).

\section{RNA preparation and quantitative real-time-polymerase chain reaction analyses}

Total RNA was extracted from both undifferentiated D3 cells or EBs at consecutive differentiation days using the MasterPure RNA Purification Kit according to the manufacturer's instructions (Epicentre Illumina, Inc.) and $2 \mu \mathrm{g}$ of RNA was reverse-transcribed into cDNA using the High Capacity RNA-to-cDNA kit (Life Technologies GmbH). Pluripotency, cardiac and germ layer marker expression was analyzed using QuantiTect Primer Assays (Qiagen $\mathrm{GmbH}$ ) (Supplementary Table S1) with Fast SYBR Green master mix (Life Technologies) to amplify the cDNA. Analyses were performed using the StepOnePlus Real-Time PCR system (Life Technologies). Relative mRNA expression was calculated from standard curves and normalized to the geometric mean of the internal reference markers Rnl8s and Gapdh.

\section{Statistics}

When applicable, data are expressed as the mean value \pm standard error of the mean or SD as indicated. In general, statistical analyses were performed using the Student's $t$-test or the Fisher's exact test and differences were considered significant if the $P$-value was $\leq 0.05\left(^{*}\right)$ or $\leq 0.01$ (**). Poissons statistics was $^{*}$ applied to calculate the error bars of the apoptosis analyses in EBs (Fig. 4), and Bernoulli statistics was applied to determine the error bars of the cumulative fraction of beating EBs (Fig. 6).

\section{Results}

Radiation alters the capacity of mouse ES cells to differentiate into spontaneously beating cardiomyocytes

To examine whether ionizing radiation influences the differentiation of D3 cells into spontaneously contracting cardiomyocytes, we applied a modified version of the EST [15]. According to the EST, contracting cardiomyocytes are determined 10 days after differentiation induction. When cells were differentiated directly after exposure (0-h scheme), a clear dose- and radiation-type-dependent reduction in the fraction of beating EBs was observed at day 10 (Fig. 1). Generally, densely ionizing $\mathrm{C}$-ions were about twice as effective as sparsely ionizing X-rays. For example, doses of $3 \mathrm{~Gy} \mathrm{C}$-ions and $6 \mathrm{~Gy}$ $\mathrm{X}$-rays were iso-effective, resulting in a beating fraction of about 2\% (Fig. 1A). When cells were differentiated $72 \mathrm{~h}$ after exposure, $\mathrm{X}$-rays were found to have no impact on the beating fraction. In contrast, a dose-dependent reduction was observed after C-ion exposure that was less severe than for EBs differentiated directly after exposure (ie, $32 \%$ compared to $2 \%$ for 3 Gy C-ions, respectively) (Fig. 1B).

To distinguish between a differentiation delay and a persistent inhibition, we extended the analysis until day 30 . Generally, the cumulative fraction of beating EBs increased with time, even in controls, in which the initial level was already high (Fig. 2). In EBs generated directly after irradiation with X-rays or C-ions, a dose-dependent delay in the appearance of beating cardiomyocyte clusters was observed (Fig. 2A, B). Yet, even for the highest doses, the cumulative fraction of beating EBs eventually approached the respective control level. Comparison of the data revealed a similar time course of beating EBs for $1 \mathrm{~Gy}$ $\mathrm{X}$-rays compared to $0.5 \mathrm{~Gy} \mathrm{C}$-ions and $6 \mathrm{~Gy} \mathrm{X}$-rays compared to $3 \mathrm{~Gy} \mathrm{C}$-ions, respectively, confirming that $\mathrm{C}$-ions are about twice as effective. However, when cells were
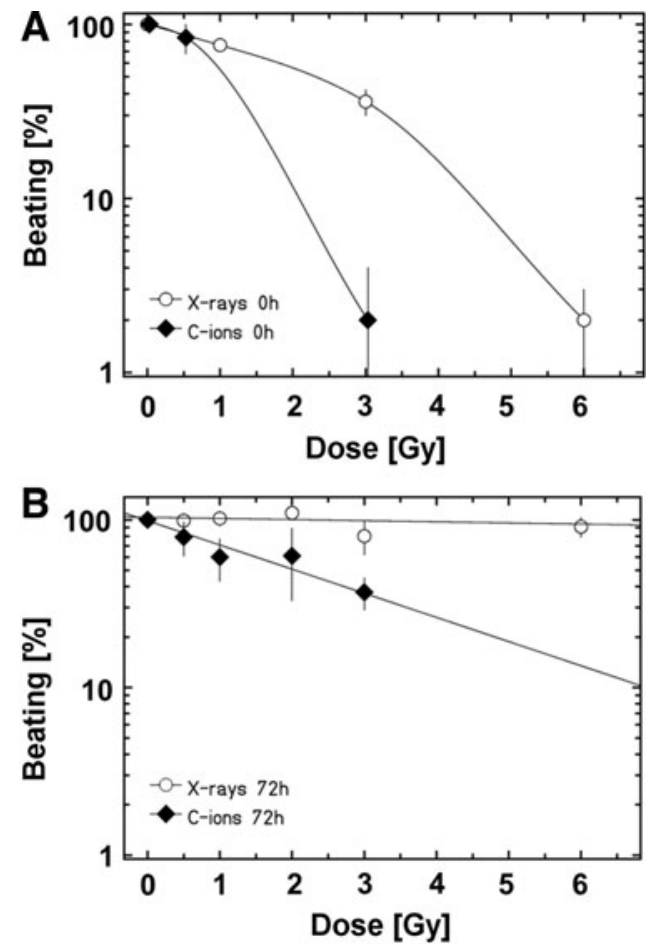

FIG. 1. Fraction of beating embryoid bodies (EBs) at day 10 of differentiation. Pluripotent D3 cells were exposed to Cions or X-rays, differentiated according to the 0 -h (A) or 72-h scheme (B), and the fraction of EBs containing spontaneously beating cardiomyocytes was determined. Percentages displayed in $\mathbf{B}$ are based on data from Luft et al. [18] with supplementations. Data points are based on 96-192 EBs from two to four independent experiments [mean $\mathrm{X} \pm$ standard error of the mean (SEM)]. 
A

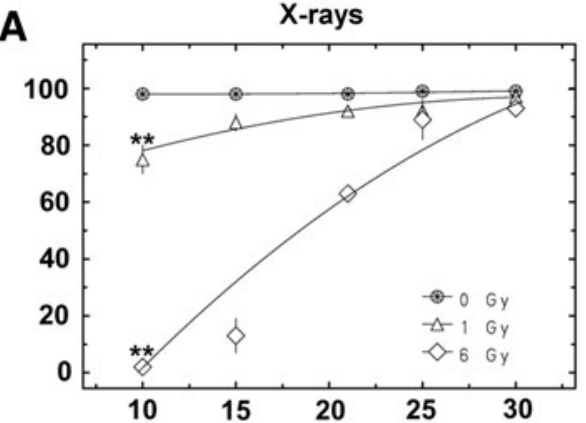

C

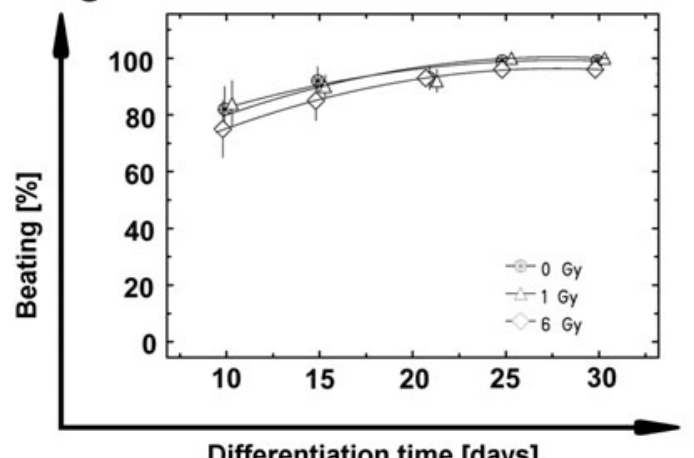

Differentiation time [days]

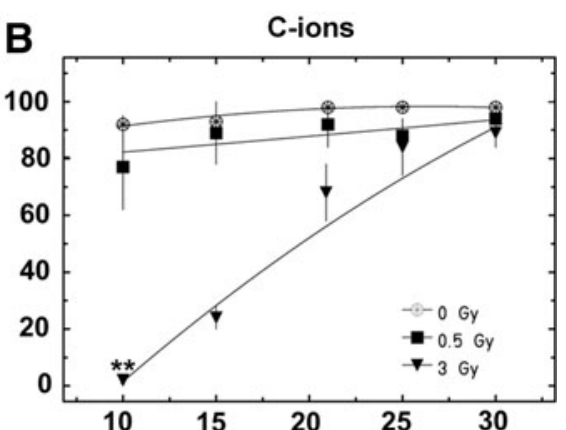

D

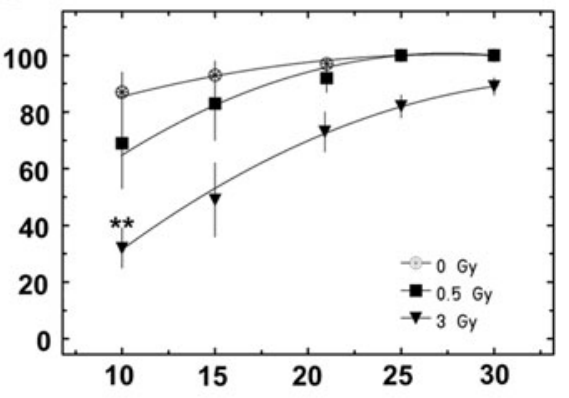

FIG. 2. Cumulative fraction of beating EBs over time. The appearance of contractile areas in the EBs was monitored from day 10 to 30 of differentiation for samples exposed to X-rays $(\mathbf{A}, \mathbf{C})$ or C-ions $(\mathbf{B}, \mathbf{D})$. Both the 0-h scheme (A, B) and the 72-h scheme (C, D) were applied. Each data point is based on 96-192 EBs from two to four independent experiments (mean $\mathrm{X} \pm \mathrm{SEM}$ ). Statistical significance was determined by the Student's $t$-test (** for $P \leq 0.01$ ). differentiated $72 \mathrm{~h}$ after irradiation, the emergence of beating clusters was delayed only by C-ions (Fig. 2C, D), indicating a more profound persistence of the damage induced by charged particles. Altogether, the data clearly show that the formation of beating cardiomyocyte clusters was considerably delayed but not permanently inhibited following radiation exposure.

\section{$E B$ size is reduced after exposure to ionizing radiation}

To elucidate, whether the EB size impacts the formation of cardiomyocytes, we measured the diameter of EBs generated from 750 nonirradiated or irradiated cells. Measurements were performed at day 5 of differentiation, when individual EBs were selected and transferred to a 24-well plate. The analysis showed that the EB size of cells irradiated with 6 Gy $\mathrm{X}$-rays or $3 \mathrm{~Gy} \mathrm{C}$-ions and processed according to the 0 -h scheme (Fig. 3A) was significantly smaller $(200 \mu \mathrm{m}$ each) than that of the respective controls $(400 \mu \mathrm{m})$. The lower doses, that is, 1 Gy X-rays and 0.5 Gy C-ions, however, did not or only slightly alter the EB size. Likewise, by applying the 72-h scheme, high-dose irradiation led to a small but significant reduction in the EB size, whereas for low doses, the difference was marginal (Fig. 3B).

\section{Radiation exposure increases the proportion of apoptotic cells}

Next, we examined if the radiation-induced reduction in EB size could be related to apoptosis. Apoptotic cells in EBs were identified based on typical changes in the nuclear morphology [21,22]. Measurements were performed at differentiation day 1,3 , and 5 . Using the 0 -h scheme, 1 day after the onset of differentiation, a dose-dependent increase
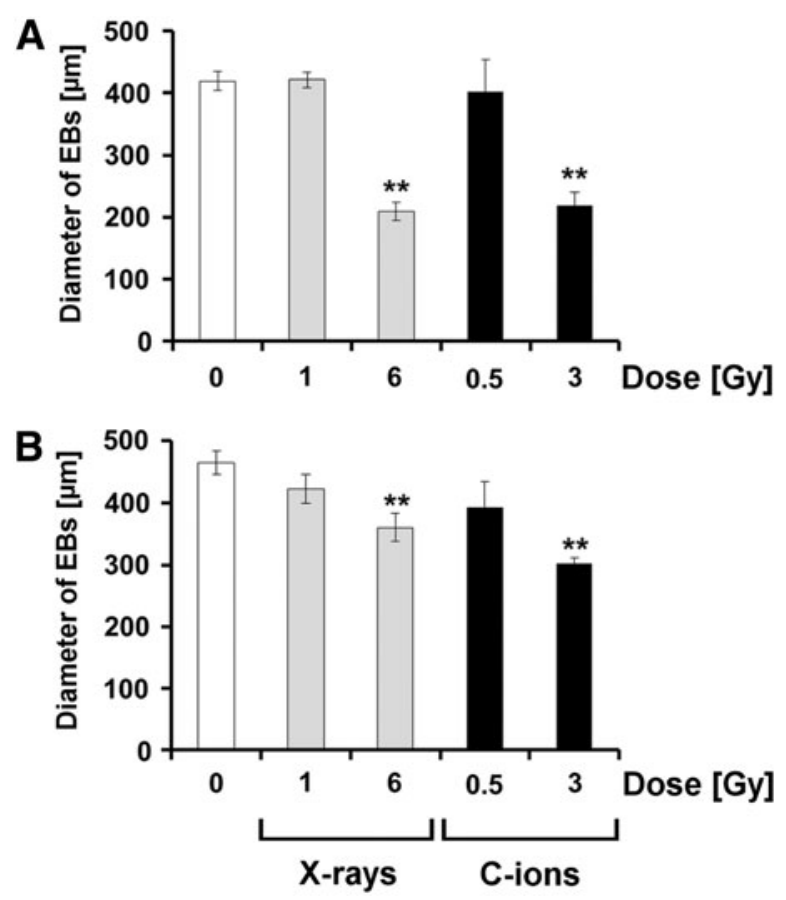

FIG. 3. Size of EBs on day 5 of differentiation. The mean diameter of EBs differentiated according to the 0-h scheme (A) and the 72-h scheme (B) from cells exposed to X-rays or C-ions was determined. Data points represent the mean $\mathrm{X} \pm \mathrm{SEM}$ for two to four independent experiments. Statistical significance was determined by the Student's $t$-test (** for $P \leq 0.01)$. 

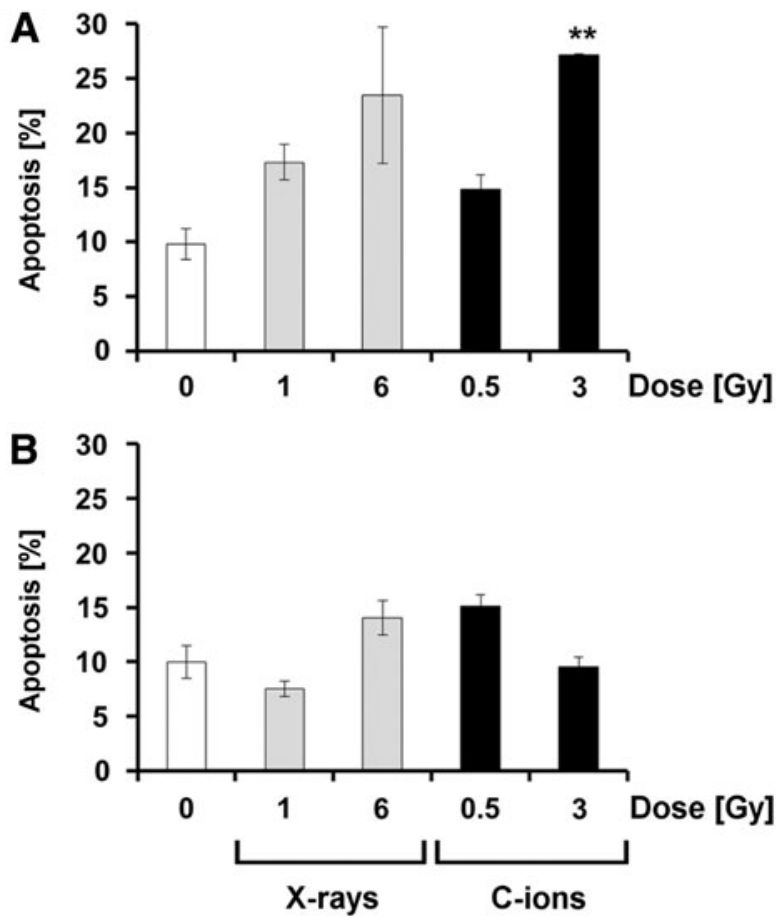

FIG. 4. Apoptosis in EBs 1 day after differentiation initiation. EBs were harvested 1 day $(\mathbf{A}, 0-\mathrm{h}$ scheme) or 4 days (B, 72-h scheme) after exposure to X-rays or C-ions. The cells' nuclear morphology was used to discriminate apoptotic from normal cells. Data points represent the mean $\mathrm{X} \pm \mathrm{SEM}$ of two to five independent experiments. Data for 0.5 and 3 Gy of $\mathrm{C}$-ions in the $72-\mathrm{h}$ scheme result from one experiment and the error bars were calculated according to Poisson statistics. Statistical significance was determined by the Student's $t$-test (** for $P \leq 0.01$ ).

in the apoptotic rate was found with $\mathrm{C}$-ions being about twice as effective as X-rays (Fig. 4A). However, only for 3 Gy C-ions, this effect was significant. At the subsequent days, apoptosis levels were generally high, ranging from $30 \%$ to $50 \%$ (data not shown), and no dose response was seen. When differentiation was induced $72 \mathrm{~h}$ after exposure, neither at day 1 (Fig. 4B) nor at day 3 or 5 (data not shown), a clear radiation effect on the apoptotic rate was visible. In line with the data obtained by the $0-\mathrm{h}$ scheme, the apoptotic levels were higher at day 3 and 5 in comparison to day 1 (data not shown). Since the radiation effects were consistently less pronounced for cells processed according to the 72-h scheme, we also measured apoptosis in irradiated D3 cultures before differentiation initiation. At day 2 and 3 after exposure to high doses (6 Gy X-rays, 3 Gy C-ions), a significantly elevated apoptotic rate was measured (Supplementary Fig. S2). Applying the low doses (1 Gy X-rays, 0.5 Gy C-ions), we found that only 1 Gy X-rays significantly increased the fraction of apoptotic cells 3 days after exposure.

\section{Clonogenic cell survival depends on dose, radiation quality, and time of plating}

To further investigate the radiosensitivity of pluripotent D3 cultures, recent measurements of the clonogenic survival after exposure to X-rays and C-ions [18] were supplemented showing excellent agreement. For both experimental con-

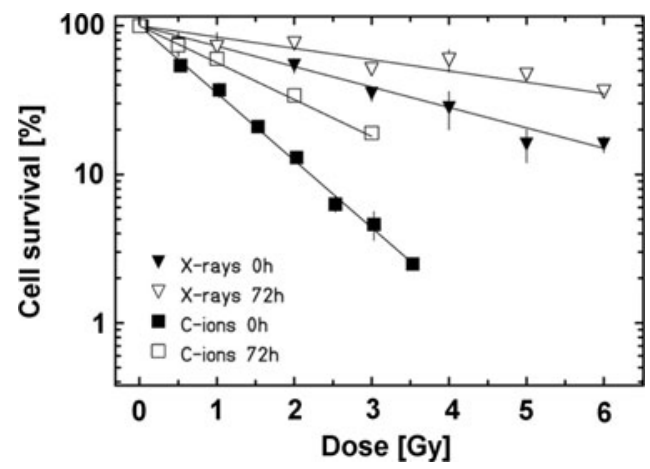

FIG. 5. Clonogenic cell survival of pluripotent D3 cells based on data from Luft et al. [18] with supplementations. Cells were plated immediately after exposure $(0 \mathrm{~h})$ or $72 \mathrm{~h}$ after exposure to $\mathrm{X}$-rays or $\mathrm{C}$-ions according to the experimental differentiation schemes. Data points represent the mean $\mathrm{X} \pm \mathrm{SD}$ for two (C-ions) and four (X-rays) experiments, respectively.

ditions (0- or 72-h scheme), the cellular survival decreased in a dose-dependent manner with $\mathrm{C}$-ions being more effective than X-rays (Fig. 5), that is, the biological effectiveness of $\mathrm{C}$-ions relative to $\mathrm{X}$-rays is about 3 for the 0 -h and $72-\mathrm{h}$ plating schemes. Thus, ionizing radiation reduces the replicating ability of ES cells in a dose- and radiation qualitydependent manner.

\section{The initial cell number in the EBs determines the onset of differentiation into cardiomyocytes}

The obtained data indicated a crucial role of the initial cell number of an EB for the formation of beating cardiomyocytes. To verify this observation, we used an increased (850 cells) or a reduced (500 cells) initial cell number for EB formation compared to the standard condition ( 750 cells). For this test, cells exposed to 3 Gy $\mathrm{X}$-rays and the respective

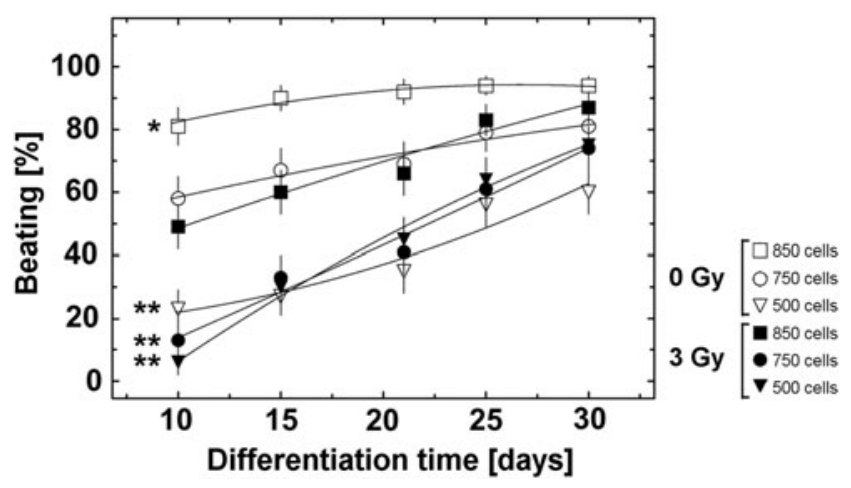

FIG. 6. Cumulative fraction of beating EBs following different initial cell numbers and exposure to X-rays. The appearance of contractile areas in the EBs was monitored from day 10 to 30 of differentiation. Cells were differentiated directly after exposure (0-h scheme) to $3 \mathrm{~Gy}$ of X-rays and EBs were produced with the initial cell number of 750 (standard conditions), 500, and 850 cells. Each data point is based on 48 EBs from one experiment. Error bars were calculated according to Bernoulli statistics. Statistical significance was determined by the Fisher's exact test for samples at day 10 compared to the control with 750 cells (* for $P \leq 0.05$, ** for $P \leq 0.01$ ). 
controls were processed according to the 0-h scheme and the fraction of beating EBs was determined. In controls, an altered number of initial cells resulted in an increased (for 850 cells) or reduced (for 500 cells) yield of beating EBs at day 10-30 when compared to standard conditions (Fig. 6). The same effect was seen after X-ray irradiation. Yet, for a given initial cell number, X-ray irradiation resulted in a lower fraction of beating EBs when compared to the nonexposed counterparts. Conversely, the radiation effect was mitigated by increasing the initial cell number. For example, at day 10, the beating fraction of EBs derived from $850 \mathrm{X}$-ray irradiated cells was close to the one derived from 750 nonexposed cells $(0.49 \pm 0.07$ and $0.58 \pm 0.07$, respectively). Thus, a clear dependency on the number of cells initially present in the EBs was demonstrated. Noteworthy, we found that the cumulative fraction of beating EBs increased persistently with time for both experimental conditions in line with the results obtained for 750 initial cells.

\section{Ionizing radiation dysregulates the expression of differentiation markers}

Exposure to ionizing radiation hampers downregulation of pluripotency markers in differentiating EBs. A delayed appearance of beating clusters after irradiation could stem from an impaired differentiation initiating capacity reflected in the persistence of pluripotency markers. Thus, the mRNA expression of crucial pluripotency factors was analyzed in samples irradiated with 3 Gy X-rays or C-ions and their respective controls at multiple differentiation stages. The expression of transcription factors such as Nanog, Oct4, and Sox2 decreased as the mouse ES cells differentiated within the EBs (Fig. 7, white columns). However, the expression of all pluripotency markers was significantly higher in samples irradiated with C-ions at day 6 and 10 of the differentiation process (Fig. 7, black columns). Expression levels in samples exposed to X-rays (Fig. 7, gray columns) were slightly higher than in the controls, but considerably lower than in C-ion irradiated cells.

In general, ionizing irradiation impairs the expression of cardiac markers. To investigate whether the delayed formation of beating cardiomyocytes was mirrored in an altered mRNA expression of markers specific for cardiac tissue formation, we performed quantitative real-time polymerase chain reaction analyses. Among the six markers studied, only the expression of $N k x 2.5$, a marker of cardiovascular progenitors, was neither affected by exposure to X-rays nor by C-ions (Fig. 8, top left panel) and peaked at day 10 of differentiation in all samples.

Unlike $N k x 2.5$, expression of Hcn4, a cyclic nucleotidegated potassium channel present in pacemaker cells and a marker of the first heart field, was significantly affected by C-ion irradiation (Fig. 8, top right panel), reaching only a third of the expression of the control at day 21, while the impact of X-rays was less severe. mRNA expression of structural and motor-regulatory factors such as cardiac actin (Actc1) and cardiac troponin $\mathrm{T}$ (Tnnt2) showed significant differences between control and irradiated samples as well (Fig. 8, middle panels). In control samples, the quantity of these transcripts increased beginning at day 21 and 25, respectively, whereas EBs generated from ES cells exposed to $\mathrm{X}$-rays and C-ions only showed increased levels at day 30.

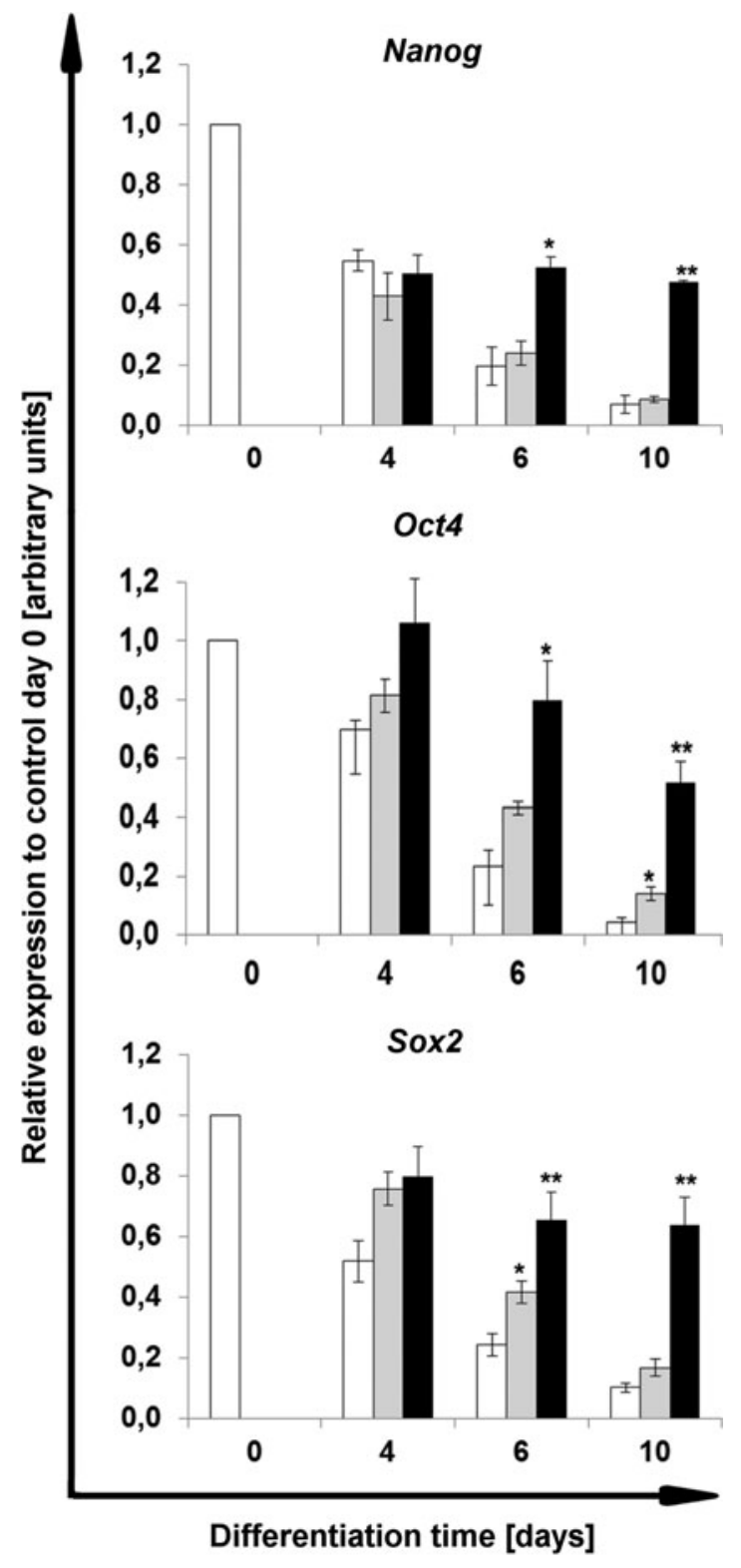

FIG. 7. Pluripotency in developing EBs is affected most severely by exposure to $\mathrm{C}$-ions. mRNA expression of pluripotency markers (Nanog, Oct4, and Sox2) was measured in samples extracted from cells at the indicated differentiation days (ranging from 0 to 15 ) after exposure to $3 \mathrm{~Gy}$ of X-rays (gray) or C-ions (black), or sham irradiated (white). Quantities are normalized to the expression levels of control cells at the day of differentiation initiation (day 0). Data points are representative of more than three independent experiments. Statistical analysis performed with Student's $t$-test showed significant differences of samples compared to day 0 control sample, $* P \leq 0.05 ; * * P \leq 0.01$.

Myosin, a motor protein, expresses specific isoforms for different cardiac areas, such as the ventricle and atrium. The heavy chain of myosin expressed in ventricles (Myh7) and the light chain expressed in the atrium (Myl4) also showed lower mRNA levels in the exposed samples compared to controls (Fig. 8, bottom panels). Similar to a delayed appearance of beating clusters, the expression of crucial cardiac differentiation markers was negatively affected by 


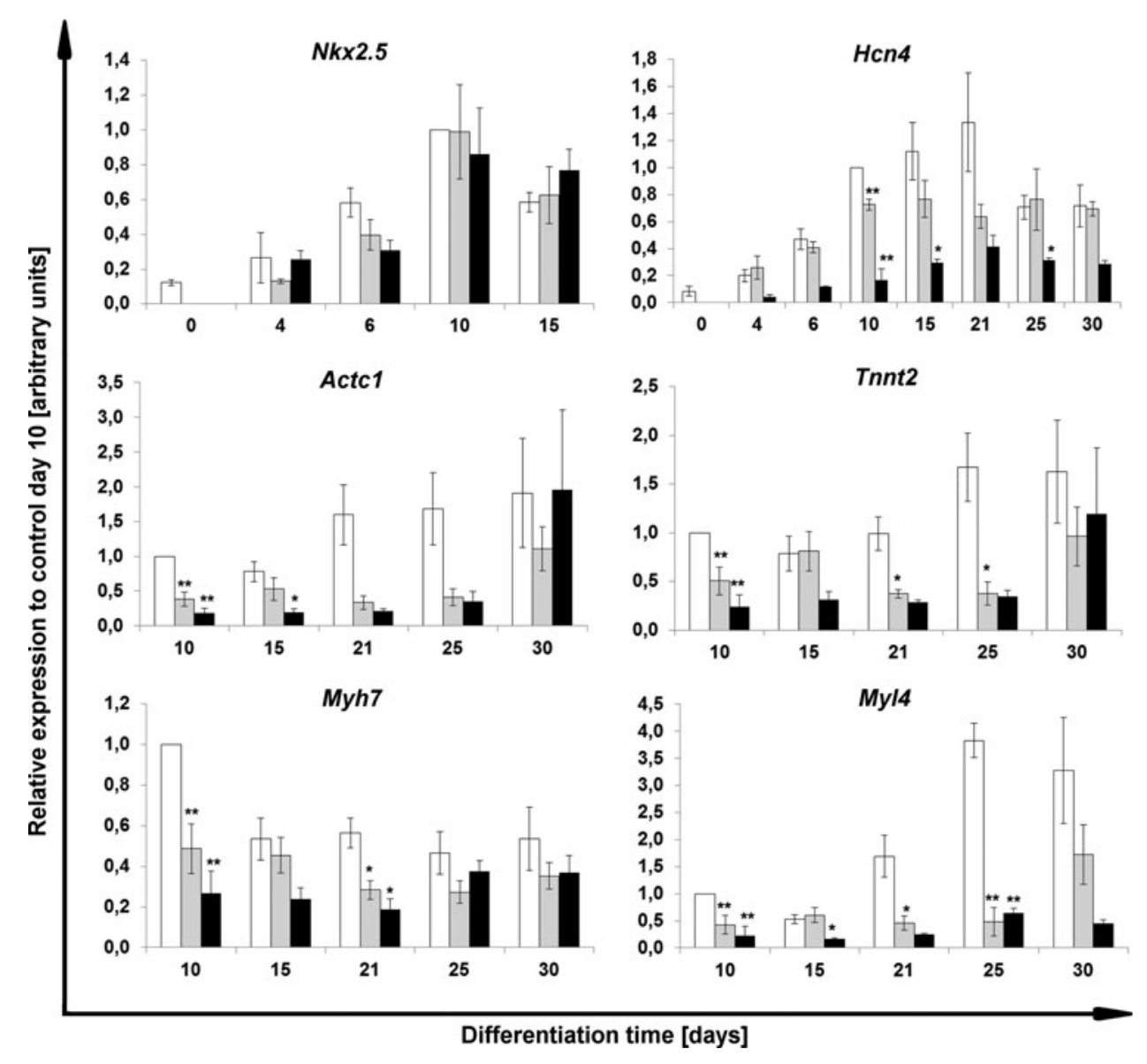

FIG. 8. Cardiac gene expression is impaired in developing EBs after exposure to $\mathrm{C}$-ions. Cardiac marker (Nkx2.5, Hcn4, Actc1, Tnnt2, Myh7, Myl4) expression levels were measured in RNA samples extracted from cells at the indicated differentiation days (ranging from 0 to 30 ) after exposure to $3 \mathrm{~Gy}$ of Xrays (gray) or $\mathrm{C}$-ions (black), or sham irradiated (white). Quantities are normalized to the expression levels of control cells at day 10 after the differentiation induction. Data points are representative of more than three independent experiments. Statistical analysis performed with Student's $t$-test showed significant differences, $* P \leq 0.05 ; * * P \leq 0.01$. exposure to ionizing radiation with $\mathrm{C}$-ions being more obstructive than X-rays. However, at day 30, most of the studied cardiac markers in X-ray- or C-ion-exposed samples reached the levels of the nonirradiated controls.

$C$-ion exposure alters the expression of germ layer markers in developing EBs. Since the delay in the initiation of cardiac marker expression could result from an altered germ layer development, specific members of each germ layer were analyzed. The mesodermal markers Brachyury $(T)$ and Crypto $(C f c 1)$, which are present in cardiac precursors, were expressed at early time points, peaking at days 4 and 6 , respectively (Fig. 9, top panels). X-ray exposure did not affect these markers, while $\mathrm{C}$-ion exposure delayed the expression of $T$ and $C f_{c} 1$. In addition, the overall levels of $T$ and more prominently of $\mathrm{Cfc} 1$ did not reach the ones of the controls.

The ectodermal markers Soxl and Nestin (Nes) also showed slight differences between control and exposed samples. Sox 1 expression peaked at day 10 in control samples, whereas X-ray- and C-ion-exposed samples showed the highest levels at day 15 (Fig. 9, middle left panel). Nestin displayed a biphasic expression pattern in control samples with an early peak at day 4 and a second burst of expression starting at day 21 (Fig. 9, middle right panel). This pattern was slightly shifted after $\mathrm{C}$-ion exposure and the second burst for $\mathrm{X}$-ray- and C-ion-exposed samples only occurred at day 30 (Fig. 9, middle right panel).

Similar to the mesoderm and ectodermal expression pattern, early endodermal markers such as Cxcr4, Foxa2, and Sox 17 showed a pronounced delay in C-ion-exposed samples compared to control samples (Supplementary Fig. S3). In contrast, when late endodermal markers (ie, for the hepatic cell lineage) such as alpha-fetoprotein $(A f p)$ or albumin $(A l b)$ were analyzed, a significant increase was observed after $\mathrm{C}$ ion irradiation (Fig. 9, bottom panels). At day 30 of differentiation, the expression of both markers was about fivefold higher in $\mathrm{C}$-ion-exposed samples compared to controls. Following X-ray irradiation, only small changes occurred compared to the controls.

Taken together, the expression of markers specific for germ layers was greatly affected by exposure to ionizing radiation. Mesodermal and early endodermal marker expression was delayed following C-ion exposure, whereas $\mathrm{X}$ ray exposure had no effect. Ectodermal marker expression was delayed for both radiation types, being slightly more pronounced for $\mathrm{C}$-ions. Interestingly, markers of the hepatic lineage were greatly induced at late time points after $\mathrm{C}$-ion irradiation.

\section{Discussion}

The current study compares the effects of sparsely and densely ionizing radiation on the early phase of embryonic development by using a validated EST [15] that is well established in pharmacological research [11] and uses the spontaneous differentiation of D3 mouse ES cells into contracting cardiomyocytes as a parameter for embryotoxicity. Mouse ES cell lines such as D3 have contributed greatly in unraveling the mechanisms of normal and pathological 


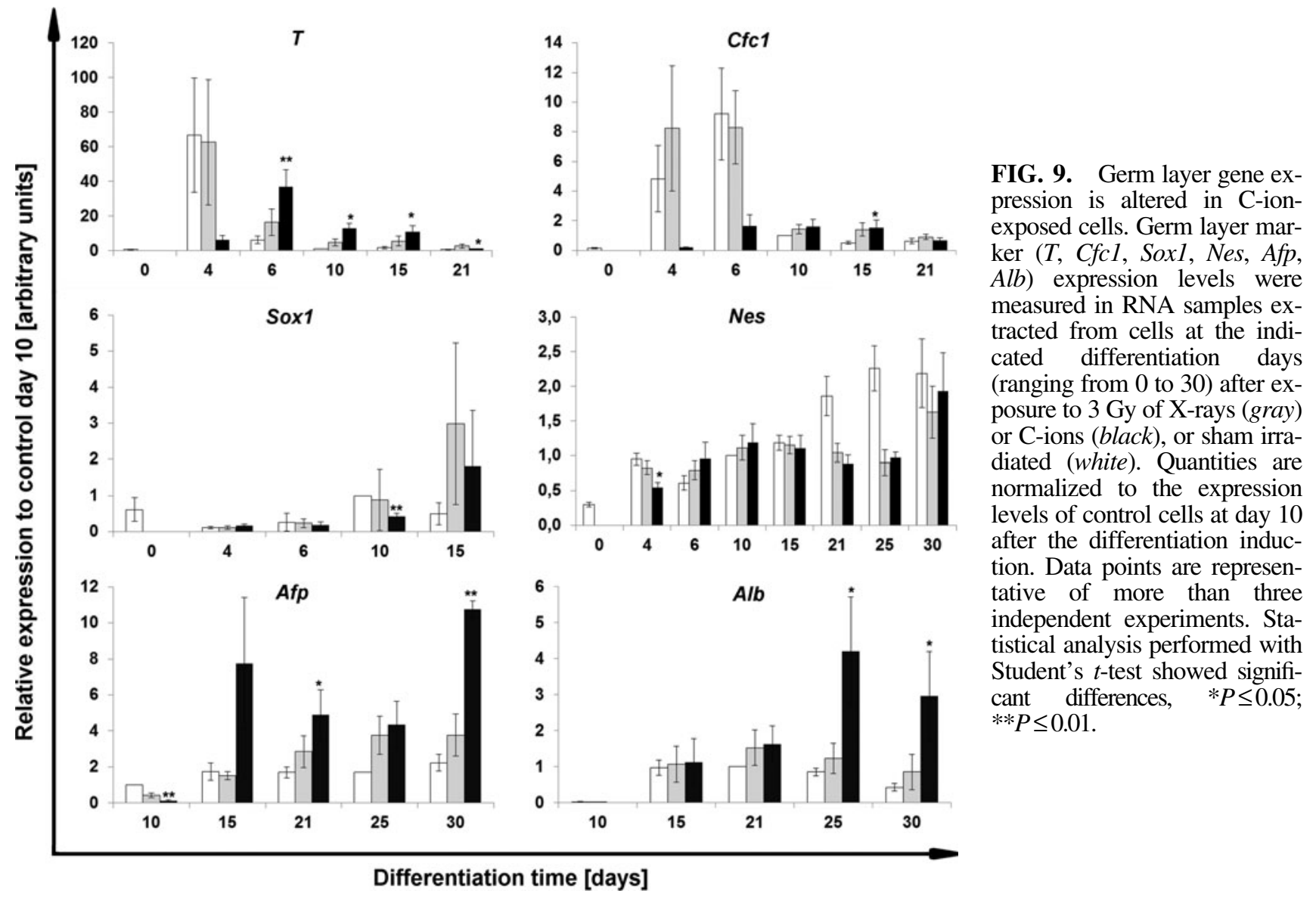

cardiac development [23] and have already been used to study the effects of sparsely ionizing radiation (eg, [18,24-26]) on embryogenesis. These studies indicate that irradiated ES cells maintain pluripotency $[18,26]$ and, upon differentiation formation, express markers of all three germ layers [26]. In a subsequent study, Rebuzzini et al. using $\gamma$-rays, found irradiated mouse ES cells to be capable of differentiating into cardiomyocytes although with altered contractile properties [27]. Less is known about the effects of densely ionizing particles on pluripotent ES cells and their differentiation capability although this radiation type is inevitably present in our environment (eg, $\alpha$-particles) and is increasingly used for therapeutic applications such as radiotherapy or radiosurgery (eg, C-ions) $[17,28,29]$ and an in utero exposure may occur due to diagnostic or therapeutic needs for the mother [30]. In a recent study, we showed that the progeny of D3 ES cells surviving the exposure to $\mathrm{C}$-ions express pluripotency markers as observed after X-ray exposure. Yet, the progeny of $\mathrm{C}$-ion irradiated cells carried significantly more chromosomal aberrations than control cultures, while an iso-dose of X-rays did not alter the aberration frequency. Furthermore, preliminary data indicate that $\mathrm{C}$-ions may impair the differentiation capability of D3 cells into cardiomyocytes [18]. In the present study, the latter aspect was further examined.

Analysis of the impact of X-rays and C-ions on D3 cells differentiated immediately after exposure (0-h scheme) showed a clear dose- and radiation-type-dependent inhibition of the formation of beating cardiomyocytes at differentiation day 10 (Fig. 1). As reported for other cellular systems, C-ions were more effective than X-rays [19,31]. Noteworthy, this inhibition did not persist. When the cells were allowed to differentiate until day 30, the beating fraction increased (Fig. 2) and finally approached the control level even after exposure to the highest doses (6 Gy X-rays or 3 Gy C-ions). Interestingly, when cells had time to recover from radiation damage before differentiation (72-h scheme), doses up to 6 Gy X-rays did not affect the formation of beating cardiomyocytes, while 3 Gy C-ions still delayed the differentiation demonstrating a sustained effect of densely ionizing radiation.

Previous studies have shown that the physical size of EBs (ie, the cell number) is a critical factor that influences the differentiation outcome [32,33], in particular cardiac development [34,35]. For example, Preda et al. reported a considerable delay in the formation of contracting areas in EBs derived from 500 murine ES cells, while no beating areas developed in EBs derived from 200 cells [35]. Accordingly, Mansergh et al. found that EBs formed by aggregation of 125-500 cells failed to express genes such as brachyury by day 4 of differentiation [33]. Brachyury in conjunction with Eomes induces the expression of MESP1 that is viewed as the "master regulator" of cardiac progenitor specification [36,37].

Thus, we measured the diameter of EBs generated from nonirradiated and irradiated cells 5 days after differentiation initiation, as displayed in Fig. 3. A significant reduction in EB size compared to the control $(P \leq 0.01)$ was solely observed after exposure to a high dose of X-rays or C-ions. In 
fact, 6 Gy X-rays and 3 Gy C-ions were iso-effective. This effect was more pronounced for EBs generated directly after exposure than for those generated $72 \mathrm{~h}$ postirradiation. For directly differentiated cells, we also found a clear relationship between reduced EB size and delayed cardiac development consistent with published data. When cells were differentiated after a recovery time (72-h scheme), the relationship between reduced EB size and delayed cardiac development was maintained following $\mathrm{C}$-ion exposure. In contrast, X-ray exposure $72 \mathrm{~h}$ before EB formation did not alter cardiac differentiation, although the EB diameter was still significantly smaller than that of the respective control (ie, $383 \pm 10 \mu \mathrm{m}$ vs. $466 \pm 11 \mu \mathrm{m}$ ). Whether the EB size represented by a diameter of about $380 \mu \mathrm{m}$ denotes the threshold for the induction of cardiac differentiation in the D3 cell line requires further investigations.

Next, we tested if an increase in the initial cell number for EB generation (ie, from 750 to 850 cells) mitigates radiation-induced delayed formation of cardiomyocytes. For practical reasons, we limited this study to cells exposed to 3 Gy X-rays and differentiated directly after exposure. This experiment showed that the fraction of beating EBs derived from 850 irradiated cells was similar to that reached by EBs derived from 750 nonirradiated cells clearly demonstrating that a certain number of initial (viable) cells is a prerequisite for a timely differentiation and that just by reducing the initial cell number (ie, to 500 cells), the fraction of beating cardiomyocytes is decreased, which is consistent with other studies (eg, [35]).

To gain insight into the processes underlying the impaired cardiac differentiation following radiation exposure, we measured the replication ability of mouse ES cells using the colony forming assay and measured apoptosis in ES cells as well as in differentiating EBs by means of morphological changes in the nuclei that accompany the programmed cell death [21]. In addition, in selected samples, caspase-3 activity, a hallmark for radiation-induced apoptosis [38], was analyzed, confirming the morphological analyses. The experiments showed that the clonogenic ability of D3 ES cells decreased with dose and that $\mathrm{C}$-ions were more effective than X-rays (Fig. 5) as observed throughout the whole study and reported by others for various rodent or human cell lines $[19,39]$. The proliferative ability of D3 cells differentiated $72 \mathrm{~h}$ postirradiation was clearly higher than for cells differentiated directly after exposure in line with an enhanced cardiac differentiation (Figs. 1 and 2).

As a reduced clonogenic ability could also be related to programmed cell death, we measured apoptosis in pluripotent D3 cells up to 3 days postirradiation (Supplementary Fig. S1). Apoptosis of pluripotent ES cells is regarded as an important mechanism to eliminate damaged cells thereby keeping the stem cell pool pristine [40,41]. Apoptosis induction in murine [26] and human ES cells [42] has already been examined following sparsely ionizing radiation. However, response of ES cells after exposure to densely ionizing radiation has not been studied yet. We found significantly elevated apoptotic rates after exposure to high doses with 6 $\mathrm{Gy} \mathrm{X}$-rays being iso-effective to $3 \mathrm{~Gy} \mathrm{C}$-ions as observed for other endpoints. Obviously, the removal of damaged cells before differentiation initiation may further contribute to impaired cardiac differentiation. Thus, we analyzed apoptosis in EBs. In EBs generated directly after exposure, dose- dependent increased apoptotic rates were found at differentiation day 1 with $\mathrm{C}$-ions being more detrimental than X-rays (Fig. 4A). In contrast, in day 1 EBs generated $72 \mathrm{~h}$ postirradiation, no significant radiation-induced increase of apoptosis was registered (Fig. 4B) underlining the pronounced effect of cell recovery before EB development. Noteworthy, in EBs at a later developmental stage (at day 3 and 5), apoptotic rates were high (30\%-50\%, data not shown) irrespective of the applied dose or differentiation scheme. Since apoptosis is essential for embryonic development (eg, for morphogenesis and cavitation) and also occurs in vitro during EB differentiation $[43,44]$, developmental apoptosis may conceal radiation-induced apoptosis. Taken together, our results obtained for cell survival and apoptosis indicate that densely ionizing radiation reduces the initial number of viable cells within an EB to a greater extent than sparsely ionizing irradiation. The reduction of the cell number subsequently leads to a reduced differentiation capability into beating cardiomyocytes as discussed earlier.

At the molecular level, the developmental delay was mirrored in an altered expression of markers for pluripotency, cardiac (mesoderm) differentiation, and differentiation into other germ layers after irradiation with 3 Gy X-rays or Cions. A clear difference in the expression of pluripotency markers between the control and C-ion irradiated samples was observed (Fig. 7). The latter maintains the expression of Nanog, Oct4, and Sox 2 even up to day 10 of differentiation. This observation is especially interesting in the case of OCT4, which has a dual function in embryogenesis. At basal levels, it binds to SOX2 to ensure pluripotency. However, increased levels of OCT4 or reduced levels of SOX2 lead to a switch toward an endodermal/mesendodermal specification as OCT4 targets SOX17 [45] that is essential for early cardiogenesis [46]. Thus, the persistent high levels of Oct4 in correlation with a delayed Sox 17 expression that only resumes to levels comparable to that of the control at day 10 hint at a greatly delayed differentiation capability of $\mathrm{C}$-ion irradiated cells toward the mesodermal lineage. These findings substantiate the data regarding the formation of beating EBs. As observed for other endpoints, X-rays were less effective.

Exposure to $\mathrm{C}$-ions generally delayed the expression of genes that mark the initiation of germ layers or are cardiac specific, as depicted in Figs. 8 and 9. Only transcription of late endodermal (liver specific) genes was greatly enhanced after C-ion exposure (Fig. 9), a phenomenon that requires further investigation. In contrast, $\mathrm{X}$-rays were usually less effective or exerted no effect at all. Similar findings were made by Rebuzzini et al. [26], who analyzed the impact of 5 Gy sparsely ionizing $\gamma$-rays on the mRNA expression of six germ layer markers during EB differentiation of R1 mouse ES cells. In line with our study that used four of the markers examined by Rebuzzini (ie, brachyury, nestin, Foxa2 and $A f p$ ), they found no significant effect of sparsely ionizing radiation compared to controls. However, in a follow-up study using the same settings, Rebuzzini et al. found an increased cardiac marker expression after sparsely ionizing radiation [27], while our results show a lower expression. Following densely ionizing $\mathrm{C}$-ion exposure, this effect was even more pronounced (Fig. 8). Whether these contradictory data are attributable to differences in the experimental design of both studies or to the cell lines used remains to be elucidated. 
In summary, the data obtained in the present study point at a delayed cardiac differentiation rather than a sustained, complete inhibition. Obviously, ES cells are able to compensate radiation-induced cell loss as proposed elsewhere [8]. However, the delay in cardiac differentiation and the obvious increased endodermal/hepatic differentiation point to a substantial dysregulation in germ layer formation. As apart from an appropriate blastocyst development, a timely regulated expression of embryonic growth factors and cytokines, controlled by a complex network of different factors is prerequisite for the implantation of the conceptus [47], a radiation-induced dysregulation of differentiation may perturb that intricate signaling and thus prevent implantation. In that context, densely ionizing $\mathrm{C}$-ions would pose a higher risk to the preimplantation embryo than sparsely ionizing radiation does due to its higher efficiency in cell killing and more severe differentiation delay. This is particularly important, because $\mathrm{C}$-ions and other charged particles are increasingly used for radiotherapy or radiosurgery.

\section{Acknowledgments}

The authors acknowledge P. Hessel for skillful assistance, S. Luft for help in flow cytometry analysis, and M. Scholz and T. Friedrich for planning and realizing the particle exposure of cells. The research leading to these results has received funding from the Euratom Seventh Framework Programme under grant agreement no. 295823 (PROCARDIO) and from the Federal Ministry of Education and Research (Bonn, Germany) under contract number 02NUK025A. A.H. was supported by HGS-HIRe. O.A. was funded by the Doctors Specialization Program of the Basque Government, Spain.

\section{Author Disclosure Statement}

No competing financial interests exist.

\section{References}

1. Scholz M. (2003). Effects of ion radiation on cells and tissues. Adv Polym Sci 162:95-156.

2. Hendry JH, SL Simon, A Wojcik, M Sohrabi, W Burkart, E Cardis, D Laurier, M Tirmarche and I Hayata. (2009). Human exposure to high natural background radiation: what can it teach us about radiation risks? J Radiol Prot 29:A29-A42.

3. Durante M and JS Loeffler. (2010). Charged particles in radiation oncology. Nat Rev Clin Oncol 7:37-43.

4. McCollough CH, BA Schueler, TD Atwell, NN Braun, DM Regner, DL Brown and AJ LeRoy. (2007). Radiation exposure and pregnancy: when should we be concerned? Radiographics 27:909-918.

5. Ratnapalan S, N Bona and G Koren. (2003). Ionizing radiation during pregnancy. Can Fam Physician 49:873-874.

6. Goldberg-Stein S, B Liu, PF Hahn and SI Lee. (2011). Body CT during pregnancy: utilization trends, examination indications, and fetal radiation doses. AJR Am J Roentgenol 196:146-151.

7. Ramanathan S and J Ryan. (2015). Radiation awareness among radiology residents, technologists, fellows and staff: where do we stand? Insights Imaging 6:133-139.

8. Streffer C, R Shore, G Konermann, A Meadows, P Uma Devi, J Preston, LE Holm, J Stather, K Mabuchi and HR Withers. (2003). Biological effects after prenatal irradiation (embryo and fetus). A report of the International Commission on Radiobiological Protection. Ann ICRP 33:5-206.

9. Schroeder IS, C Wiese, TT Truong, A Rolletschek and AM Wobus. (2009). Differentiation analysis of pluripotent mouse embryonic stem (ES) cells in vitro. In: Gene Knockout Protocols. Kuehn R and W Wurst, eds. Humana Press, a part of Springer Science \& Business Media, New York, pp 219-250.

10. Genschow E, H Spielmann, G Scholz, A Seiler, N Brown, A Piersma, M Brady, N Clemann, H Huuskonen, et al. (2002). The ECVAM international validation study on in vitro embryotoxicity tests: results of the definitive phase and evaluation of prediction models. European Centre for the Validation of Alternative Methods. Altern Lab Anim 30:151-176.

11. Wobus AM and P Löser. (2011). Present state and future perspectives of using pluripotent stem cells in toxicology research. Arch Toxicol 85:79-117.

12. Abassi YA, B Xi, N Li, W Ouyang, A Seiler, M Watzele, R Kettenhofen, H Bohlen, A Ehlich, et al. (2012). Dynamic monitoring of beating periodicity of stem cell-derived cardiomyocytes as a predictive tool for preclinical safety assessment. Br J Pharmacol 165:1424-1441.

13. Doetschman TC, H Eistetter, M Katz, W Schmidt and R Kemler. (1985). The in vitro development of blastocystderived embryonic stem cell lines: formation of visceral yolk sac, blood islands and myocardium. J Embryol Exp Morphol 87:27-45.

14. Van Laake LW, D Van Hoof and CL Mummery. (2005). Cardiomyocytes derived from stem cells. Ann Med 37: 499-512.

15. Seiler AEM and H Spielmann. (2011). The validated embryonic stem cell test to predict embryotoxicity in vitro. Nat Protoc 6:961-978.

16. Buesen R, E Genschow, B Slawik, A Visan, H Spielmann, A Luch and A Seiler. (2009). Embryonic stem cell test remastered: comparison between the validated EST and the new molecular FACS-EST for assessing developmental toxicity in vitro. Toxicol Sci 108:389-400.

17. Loeffler JS and M Durante. (2013). Charged particle therapy-optimization, challenges and future directions. Nat Rev Clin Oncol 10:411-424.

18. Luft S, D Pignalosa, E Nasonova, O Arrizabalaga, A Helm, M Durante and S Ritter. (2014). Fate of D3 mouse embryonic stem cells exposed to X-rays or carbon ions. Mutat Res Genet Toxicol Environ Mutagen 760:56-63.

19. Weyrather WK, S Ritter, M Scholz and G Kraft. (1999). RBE for carbon track-segment irradiation in cell lines of differing repair capacity. Int J Radiat Biol 75:1357-1364.

20. Franken NAP, HM Rodermond, J Stap, J Haveman and C van Bree. (2006). Clonogenic assay of cells in vitro. Nat Protoc 1:2315-2319.

21. Johnson VL, SCW Ko, TH Holmstrom, JE Eriksson and SC Chow. (2000). Effector caspases are dispensable for the early nuclear morphological changes during chemicalinduced apoptosis. J Cell Sci 113:2941-2953.

22. Meijer AE, U-SE Kronqvist, R Lewensohn and M HarmsRingdahl. (1998). RBE for the induction of apoptosis in human peripheral lymphocytes exposed in vitro to highLET radiation generated by accelerated nitrogen ions. Int $\mathbf{J}$ Radiat Biol 73:169-177.

23. Van Vliet P, SM Wu, S Zaffran and M Pucéat. (2012). Early cardiac development: a view from stem cells to embryos. Cardiovasc Res 96:352-362. 
24. Thomas JW, C LaMantia and T Magnuson. (1998). X-rayinduced mutations in mouse embryonic stem cells. Proc Natl Acad Sci U S A 95:1114-1119.

25. Denissova NG, IV Tereshchenko, E Cui, PJ Stambrook, C Shao and JA Tischfield. (2011). Ionizing radiation is a potent inducer of mitotic recombination in mouse embryonic stem cells. Mutat Res 715:1-6.

26. Rebuzzini P, D Pignalosa, G Mazzini, R Di Liberto, A Coppola, N Terranova, P Magni, CA Redi, M Zuccotti and S Garagna. (2012). Mouse embryonic stem cells that survive $\gamma$ rays exposure maintain pluripotent differentiation potential and genome stability. J Cell Physiol 227:1242-1249.

27. Rebuzzini P, L Fassina, F Mulas, R Bellazzi, CA Redi, R Di Liberto, G Magenes, J Adjaye, M Zuccotti and S Garagna. (2013). Mouse embryonic stem cells irradiated with $\gamma$-rays differentiate into cardiomyocytes but with altered contractile properties. Mutat Res 756:37-45.

28. Schardt D, T Elsässer and D Schulz-Ertner. (2010). Heavyion tumor therapy: physical and radiobiological benefits. Rev Mod Phys 82:383-425.

29. Bert C, R Engenhart-Cabillic and M Durante. (2012). Particle therapy for noncancer diseases. Med Phys 39:1716-1727.

30. Münter MW, M Wengenroth, G Fehrenbacher, D Schardt, A Nikoghosyan, M Durante and J Debus. (2010). Heavy ion radiotherapy during pregnancy. Fertil Steril 94:2329.e5-e7.

31. Hartel C, A Nikoghosyan, M Durante, S Sommer, E Nasonova, C Fournier, R Lee, J Debus, D Schulz-Ertner and S Ritter. (2010). Chromosomal aberrations in peripheral blood lymphocytes of prostate cancer patients treated with IMRT and carbon ions. Radiother Oncol 95:73-78.

32. Messana JM, NS Hwang, J Coburn, JH Elisseeff and Z Zhang. (2008). Size of the embryoid body influences chondrogenesis of mouse embryonic stem cells. J Tissue Eng Regen Med 2:499-506.

33. Mansergh FC, CS Daly, AL Hurley, MA Wride, SM Hunter and MJ Evans. (2009). Gene expression profiles during early differentiation of mouse embryonic stem cells. BMC Dev Biol 9:5.

34. Choi YY, BG Chung, DH Lee, A Khademhosseini, J-H Kim and S-H Lee. (2010). Controlled-size embryoid body formation in concave microwell arrays. Biomaterials 31:42964303.

35. Preda M, A Burlacu and M Simionescu. (2013). Defined-size embryoid bodies formed in the presence of serum replacement increases the efficiency of the cardiac differentiation of mouse embryonic stem cells. Tissue Cell 45:54-60.

36. Bondue A, G Lapouge, C Paulissen, C Semeraro, M Iacovino, M Kyba and C Blanpain. (2008). Mesp1 acts as a master regulator of multipotent cardiovascular progenitor specification. Cell Stem Cell 3:69-84.

37. Burridge PW, G Keller, JD Gold and JC Wu. (2012). Production of de novo cardiomyocytes: human pluripotent stem cell differentiation and direct reprogramming. Cell Stem Cell 10:16-28.
38. Verheij M and H Bartelink. (2000). Radiation-induced apoptosis. Cell Tissue Res 301:133-142.

39. Habermehl D, K Ilicic, S Dehne, S Rieken, L Orschiedt, S Brons, T Haberer, KJ Weber, J Debus and SE Combs. (2014). The relative biological effectiveness for carbon and oxygen ion beams using the raster-scanning technique in hepatocellular carcinoma cell lines. PLoS One 9: e113591.

40. Hong Y and PJ Stambrook. (2004). Restoration of an absent G1 arrest and protection from apoptosis in embryonic stem cells after ionizing radiation. Proc Natl Acad Sci U S A 101:14443-14448.

41. Tichy ED. (2011). Mechanisms maintaining genomic integrity in embryonic stem cells and induced pluripotent stem cells. Exp Biol Med 236:987-996.

42. Wilson KD, N Sun, M Huang, WY Zhang, AS Lee, Z Li, SX Wang and JC Wu. (2010). Effects of ionizing radiation on self renewal and pluripotency of human embryonic stem cells. Cancer Res 70:5539-5548.

43. Karbanová J and J Mokrý. (2002). Histological and histochemical analysis of embryoid bodies. Acta Histochem 104: 361-365.

44. Joza N, SA Susin, E Daugas, WL Stanford, SK Cho, CYJ Li, T Sasaki, AJ Elia, HM Cheng, et al. (2001). Essential role of the mitochondrial apoptosis-inducing factor in programmed cell death. Nature 410:549-554.

45. Stefanovic S, N Abboud, S Désilets, D Nury, C Cowan and M Pucéat. (2009). Interplay of Oct4 with Sox2 and Sox17: a molecular switch from stem cell pluripotency to specifying a cardiac fate. J Cell Biol 186:665-673.

46. Liu Y, M Asakura, H Inoue, T Nakamura, M Sano, Z Niu, M Chen, RJ Schwartz and MD Schneider. (2007). Sox17 is essential for the specification of cardiac mesoderm in embryonic stem cells. Proc Natl Acad Sci U S A 104: 3859-3864.

47. Singh M, P Chaudhry and E Asselin. (2011). Bridging endometrial receptivity and implantation: network of hormones, cytokines, and growth factors. J Endocrinol 210: 5-14.

Address correspondence to: Sylvia Ritter

Biophysics Department GSI Helmholtz Centre for Heavy Ion Research Planckstrasse 1 Darmstadt 64291

Germany

E-mail: s.ritter@gsi.de

Received for publication August 12, 2015 Accepted after revision October 27, 2015 Prepublished on Liebert Instant Online October 28, 2015 